\title{
Psoas Muscle Hydatid Cyst Causing Ureteric Compression and Hydronephrosis
}

\author{
Riyaz Ahmad Sheikh ${ }^{1}$, Rayees Ahmad Dar ${ }^{\text {* }}$, Sabiya Hamid Wani ${ }^{2}$, Mushtaq Ahmad Gagloo', \\ Suhail Farooq Mir ${ }^{1}$, Gulzar Ahmad Bhat ${ }^{1}$, Irfan Jan Khan ${ }^{1}$ \\ ${ }^{1}$ Department of General Surgery, Sheri Kashmir Institute of Medical Sciences, Srinagar, India; ${ }^{2}$ Department of Surgery, Government \\ Medical College, Srinagar, India. \\ Email: *dr.rayeesdar@gmail.com
}

Received March $10^{\text {th }}, 2012$; revised April 14 ${ }^{\text {th }}, 2012$; accepted May $4^{\text {th }}, 2012$

\begin{abstract}
Psoas muscle is a rare location for hydatid disease. Here we present a case of infected hydatid cyst left psoas muscle presenting as left flank pain, fever, local flank tenderness, and raised blood counts. Pre-operative diagnosis was made by Ultrasonography (USG) and Computed Tomography (CT) abdomen, although serology for hydatid disease was negative.
\end{abstract}

Keywords: Hydatid Cyst; Psoas Muscle; Hydronephrosis

\section{Introduction}

Hydatid disease is a zoonotic disease caused by Echinococcus granulosus. Man is an accidental intermediate host while animals can be both definitive as well as intermediate host. In human 50\% - 75\% cysts are present in liver, $25 \%$ in the lungs and the rest in various tissues of the body. $2 \%$ - 3\% of hydatid cysts occur in muscular tissue [1].

\section{Case Report}

A 35-year-old female patient presented to our emergency department with complaints of left flank pain, fever and few episodes of vomiting from last 3 days. On general physical examination, patient was having tachycardia with pulse rate of 108 beats per minute, and temperature was 103 degree Fahrenheit. Local examination revealed tender left flank region. Complete blood count revealed total leukocyte count of 18,000 per cubic millimeter, with neutrophils constituting $87 \%$ of cell count. USG abdomen showed a retroperitoneal cystic lesion infero-medial to left kidney causing significant mass effect on the kidney (Figure 1). Contrast enhanced CT scan abdomen showed a cystic lesion $9 \times 6 \times 6 \mathrm{~cm}$ infero-medial to left kidney which was hydronephrotic. Significant mass effect at renal hilum was seen (Figure 2). Ureter was also displaced laterally. Serology for hydatid disease was sent which, however, was not suggestive. Medical therapy was started will intravenous (IV) antibiotics, fluids and

*Corresponding author. analgesics. But patient did not improve and it was decided to explore the patient. Abdomen was opened by classical left flank incision, and retroperitoneal space entered. A large $9 \times 7 \times 7 \mathrm{~cm}$ infected hydatid cyst (Figure 3) was found adjacent to inferior pole of left kidney, obstructing upper part of ureter and displacing inferior pole of kidney. After the operative field was protected with swabs soaked with $3 \%$ cetrimide, the cyst was excised. The cyst was full of pus with some membrane like debris. The cyst along with the infected material was sent for analysis which confirmed hydatid disease. Post-operative period was uneventful and patient was discharged on fifth post-operative day on oral Albendazole.

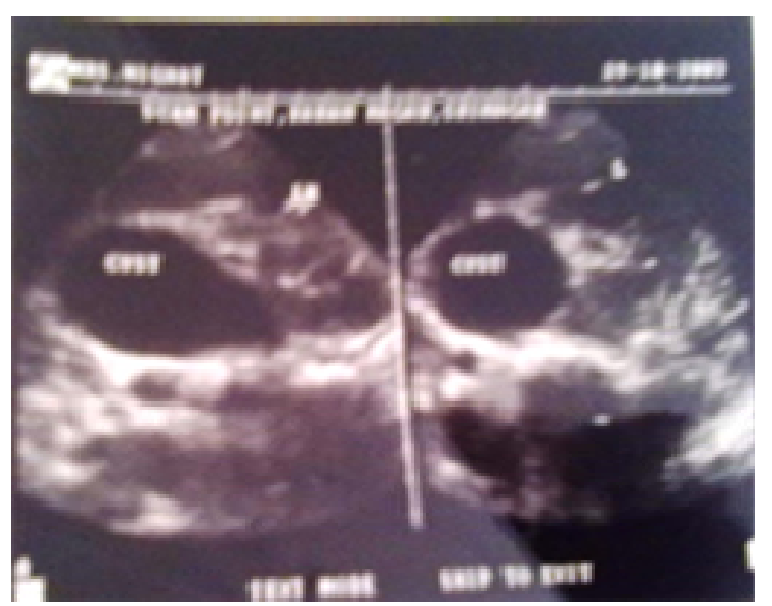

Figure 1. USG abdomen showing a retroperitoneal cystic lesion infero-medial to left kidney. 

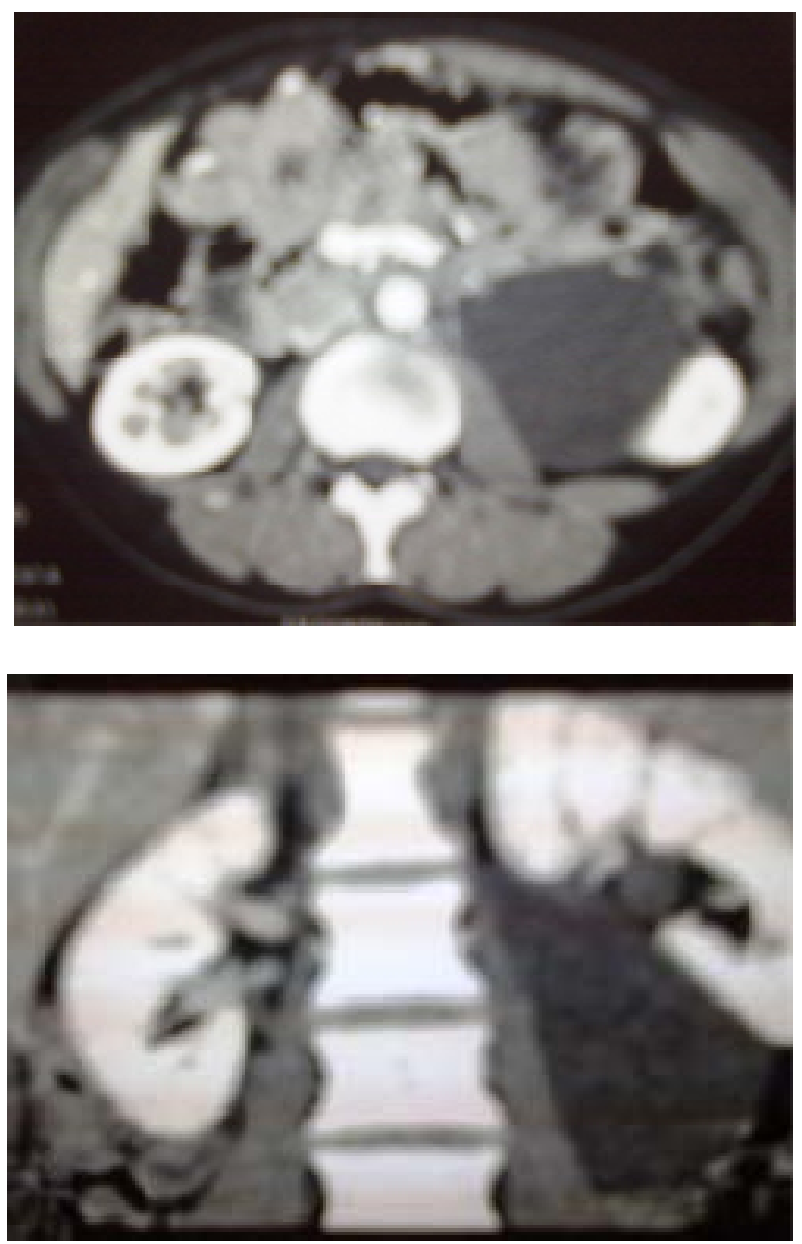

Figure 2. Contrast enhanced CT scan abdomen (Axial and Coronal cuts) showing a cystic lesion infero-medial to left hydronephrotic kidney. Significant mass effect at renal hilum is seen.

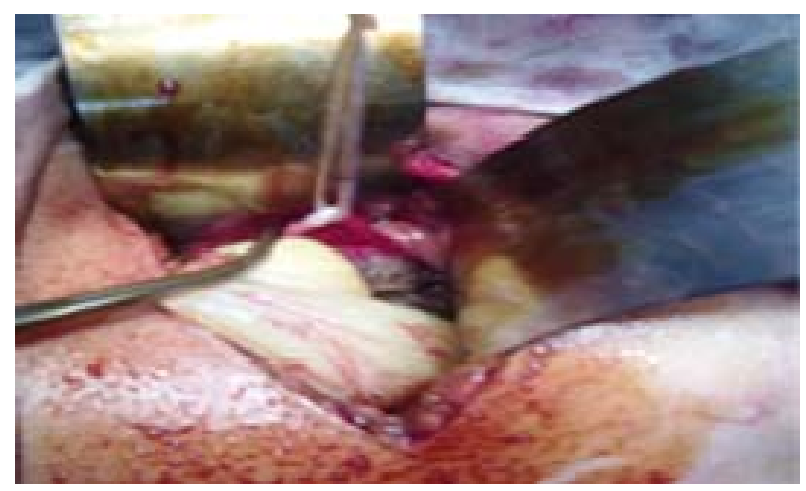

Figure 3. Intraoperative view of cyst containing membrane like material.

\section{Discussion}

Hydatid cyst can occur in any part of body. When the hydatid cyst is located in the muscle the diagnosis of the cyst may be difficult and late, because this location is unexpected and the cyst is asymptomatic unless it enlarges and compresses adjacent organs. Although the skin and serological test are widely used to confirm diagnosis, they are negative because the parasite is isolated by the capsule from the immune system. Hydatid cyst is rarely located in psoas muscle and if it does not compress kidney, ureter or vertebra, it remains asymptomatic and may be diagnosed incidentally. Radiological investigations [USG and CT abdomen] are used to establish the diagnosis and to find the extent of disease. Recently, MRI is gaining place in the diagnosis of hydatid cyst of soft tissue [2]. Cystic and complex retroperitoneal tumor, pyogenic abscess of psoas muscle and even tuberculosis of psoas must be considered in the differential diagnosis of psoas hydatid [3]. After pre-operative diagnosis of hydatid cyst, adequate excision of the cyst with sterilization of the cyst cavity by scolicidal agents should be done. Medical therapy with Mebendazole or Albendazole is used for prophylaxis and postoperatively to prevent the recurrence. Pre-operative medical therapy should be started at least 4 days before surgery and continued for one month or preferably several months after surgery [4,5]. Extra-peritoneal approach is preferred in order to prevent dissemination into peritoneal cavity. In summary, the case presented here is notable because of the rarity of the disease and because of the cyst causing compression of the ureter with displacement and hydronephrosis of kidney.

\section{REFERENCES}

[1] M. Marongiut, F. Santu, et al., "Primary Hydatidosis of Psoas Muscle,” ANZ Journal of Surgery, Vol. 27, 2002, pp. 443-445.

[2] B. Claikens, L. Van Hoe and W. van Steenbergen, "Images in Clinical Research, MRI Features of Hydatid Disease,” Belgian Journal of Radiology, Vol. 82, 1999, pp. 167-169.

[3] J. C. Angulo, J. Granell, J. Muguerza and M. Sanchez-Chapado, "Primary Bilateral Hydatidosis of Psoas Muscle,” Journal of Urology, Vol. 161, No. 5, 1999, pp. 1557-1558. doi:10.1016/S0022-5347(05)68953-7

[4] M. Morovic, "Human Hydatidosis in Dalmatia, Croatia," Epidemiology \& Infection, Vol. 119, No. 2, 1997, pp. 271-276.

[5] I. Glunoo, Rojez, N. Bradanc, A. Petricevic, V. P. Pisac and V. Glunac, "Primary Echinococossis of Sternocleidomastoid,” Croatian Medical Journal, Vol. 42, 2001, pp. 196-198. 